\title{
Orientation mapping of semicrystalline polymers using scanning electron nanobeam diffraction
}

\author{
Ouliana Panova $^{1,2}$, X. Chelsea Chen ${ }^{3,4}$, Karen C. Bustillo ${ }^{2}$, Colin Ophus ${ }^{2}$, Mahesh P. Bhatt ${ }^{4,5}$, Nitash \\ Balsara $^{3,4,5}$, Andrew M. Minor ${ }^{1,2,6}$ \\ ${ }^{1}$ Department of Materials Science and Engineering, University of California, Berkeley, CA, USA \\ ${ }^{2}$ National Center for Electron Microscopy, Molecular Foundry, Lawrence Berkeley National Laboratory, Berkeley, \\ CA, USA \\ ${ }^{3}$ Department of Chemical and Biomolecular Engineering, University of California, Berkeley, CA, USA \\ ${ }^{4}$ Materials Science Division, Lawrence Berkeley National Laboratory, Berkeley, CA, USA \\ ${ }^{5}$ Joint Center for Energy Storage Research, Lawrence Berkeley National Laboratory, Berkeley, CA, USA \\ ${ }^{6}$ Corresponding author: aminor@1bl.gov
}

KEYWORDS: TEM, STEM, spatially resolved, diffraction, crystal orientation, P3HT, polymers, locally resolved structure

\begin{abstract}
We demonstrate a scanning electron nanobeam diffraction technique that can be used for mapping the size and distribution of nanoscale crystalline regions in a polymer blend. In addition, it can map the relative orientation of crystallites and the degree of crystallinity of the material. The model polymer blend is a 50:50 w/w mixture of semicrystalline poly(3-hexylthiophene-2,5-diyl) (P3HT) and amorphous polystyrene (PS). The technique uses a scanning electron beam to raster across the sample and acquires a diffraction image at each probe position. Through image alignment and filtering, the diffraction image dataset enables mapping of the crystalline regions within the scanned area and construction of an orientation map.
\end{abstract}

\section{Introduction}

An outstanding question in the field of semicrystalline polymer characterization is how to directly observe the nanoscale orientation of crystalline domains. While characterization techniques such as electron backscattered scanning diffraction (Randle, 2009) can efficiently map the local grain structure in metals and ceramics at the nanometer scale, the sensitivity of polymer structure to electron bombardment precludes the use of these techniques, which require an extended exposure or a high electron dose. Furthermore, soft materials usually consist of light elements (e.g. C, O, N, and H) and lack contrast across different nanostructures as well as between nanostructures and the matrix. Most importantly, due to their chain folding mechanism, crystalline regions in a polymer are always surrounded by amorphous regions, which leads to local variations in the degree of crystallinity.

A number of characterization techniques have been successful in providing structural information in soft materials: X-ray diffraction (Kadem et al., 2014; Park et al., 2005), small-angle X-ray scattering (Dudenko et al., 2012; Kao et al., 2014; Mårdalen et al., 1992), wide-angle X-ray scattering (Duong et al., 2013; Müller et al., 2008; Perlich et al., 2010; Xiao et al., 2010), differential scanning calorimetry (Chan et al., 2010; da Silva and Mortimer, 1984; Remy et al., 2014), nuclear magnetic resonance imaging (Yazawa et al., 2010), UV-vis spectroscopy (Hammer et al., 2014), and molecular simulation 
models (Meredig et al., 2009). However, these techniques typically furnish data that is averaged over the bulk of the sample and thus do not provide a map of the dispersion and relative orientation of the crystallites within a local area. A few spatially resolved techniques have shown to be effective in mapping local polymer structure: a high-resolution transmission electron microscopy (TEM) technique has mapped polymer structure by tracing the lattice fringe arrangement visible in bright field (Takacs et al., 2014; Takacs et al., 2013); electron energy-loss spectroscopy spectra have been used in conjunction with scanning transmission electron microscopy (STEM) to differentiate between polymer molecules by their bonding types (Siangchaew et al., 1996); and cryo-TEM tomography has determined the location of crystalline regions in polymers via diffraction of the encasing ice (Wirix et al., 2014).

The nanoscale orientation of semicrystalline polymers directly affects their electronic properties (Chu et al., 2008; Coropceanu et al., 2007), including their ability to generate excitons (Paquin et al., 2015) or carry charge (McMahon et al., 2011). While they do not yet rival the efficiency of ceramic materials, organic polymers and blends are promising candidates for organic photovoltaic (OPV) applications (Mazzio and Luscombe, 2014) due to their portability, low cost and ease of manufacturing. The preferential crystallization of poly(3-hexylthiopene)(P3HT)(Ludwigs et al., 2014) along its pi-pi bonds (Northrup, 2007) allows electrons and holes to easily move within the crystal bulk (Dag and Wang, 2010), which has a direct effect on device efficiency. P3HT was chosen as the subject of study in this work because its bulk and nanoscale crystalline structures have been examined extensively. In order to study distinct morphologies, the P3HT was blended with polystyrene (PS), which is known to form a phase-separated morphology with distinct phase boundaries that facilitated the validation of our image reconstruction methods. This work addresses the size, distribution, and the local Bragg peak orientation of crystalline domains in $\mathrm{P} 3 \mathrm{HT}$, information that is critical for engineering the improvement of organic photovoltaic materials.

In this study it is our goal to introduce a new technique for imaging semicrystalline polymers on the nanoscale; we apply this technique to the technologically relevant P3HT to produce spatially resolved maps of the crystalline domains.

\section{Experimental methods}

\section{a. Materials and techniques}

Regioregular P3HT was synthesized (93\% regioregularity), purified, and characterized using the techniques described by Bhatt et al (Bhatt et al., 2015). The molecular weights of P3HT and PS used in this work were $9 \mathrm{~kg} / \mathrm{mol}$ and $10.8 \mathrm{~kg} / \mathrm{mol}$, respectively. P3HT and PS were dissolved separately in a solution of chlorobenzene (Motaung et al., 2010; Xie et al., 2009) with a concentration of $10 \mathrm{mg} / \mathrm{mL}$ and subsequently filtered with $0.2 \mu \mathrm{m}$ polytetrafluoroethylene filters. A 50:50 w/w blend of P3HT and PS was made by blending equal weight of P3HT and PS solutions. The mixed solution was spin-coated onto a glass substrate at $3000 \mathrm{rpm}$ for $60 \mathrm{~s}$, resulting in a $30 \mathrm{~nm}$ thick film which was then deposited onto a lacey carbon-supported copper grid via lift-off in deionized water. The TEM samples were dried at room temperature in vacuum for 24 hours and subsequently annealed for 1 hour at $150^{\circ} \mathrm{C}$ in an Ar-filled glovebox.

The 4-dimensional scanning electron microscopy (4D-STEM) data was acquired using a FEI Titan TEM operating at 200 and $300 \mathrm{kV}$ (Figure 1). A custom written Python script directed the STEM acquisition, which used a convergent beam with a convergence half-angle of $0.51 \mathrm{mrad}$ (Figure 1a) to raster over an $m \times n$ area and collect a stack of diffraction patterns (Figure 1b). While the convergent probe allowed for STEM rastering, the small convergence angle ensured that the diffraction disks did not overlap in the diffraction patterns (DPs). The probe in this study was not aberration corrected. Initial low-dose low-mag STEM images (dwell time of $1 \mu \mathrm{s}$ ) enabled us to find the regions of interest. Annular dark-field STEM images were collected with a semi-angle of $34 \mathrm{mrad}$. The rastering was performed with 
a 20 and $40 \mathrm{~nm}$ step size and an exposure of 70 and $50 \mathrm{~ms}$ respectively; the time was chosen to optimize the diffracted signal given a chosen probe current. The $20 \mathrm{~nm}$ minimum step size was chosen because it was the smallest step size that did not result in a decrease in the Bragg scattered peak intensities. The diffraction images were captured with a Gatan Orius camera, but a more sensitive camera might be able to record similar diffraction intensities with a shorter dwell time and electron dose, effectively increasing the spatial resolution of the technique. The presence of sulfur atoms within P3HT backbone allowed us to map the sulfur K-edge via electron dispersive spectroscopy (EDS) in the TEM to confirm the presence and distribution of P3HT molecules.

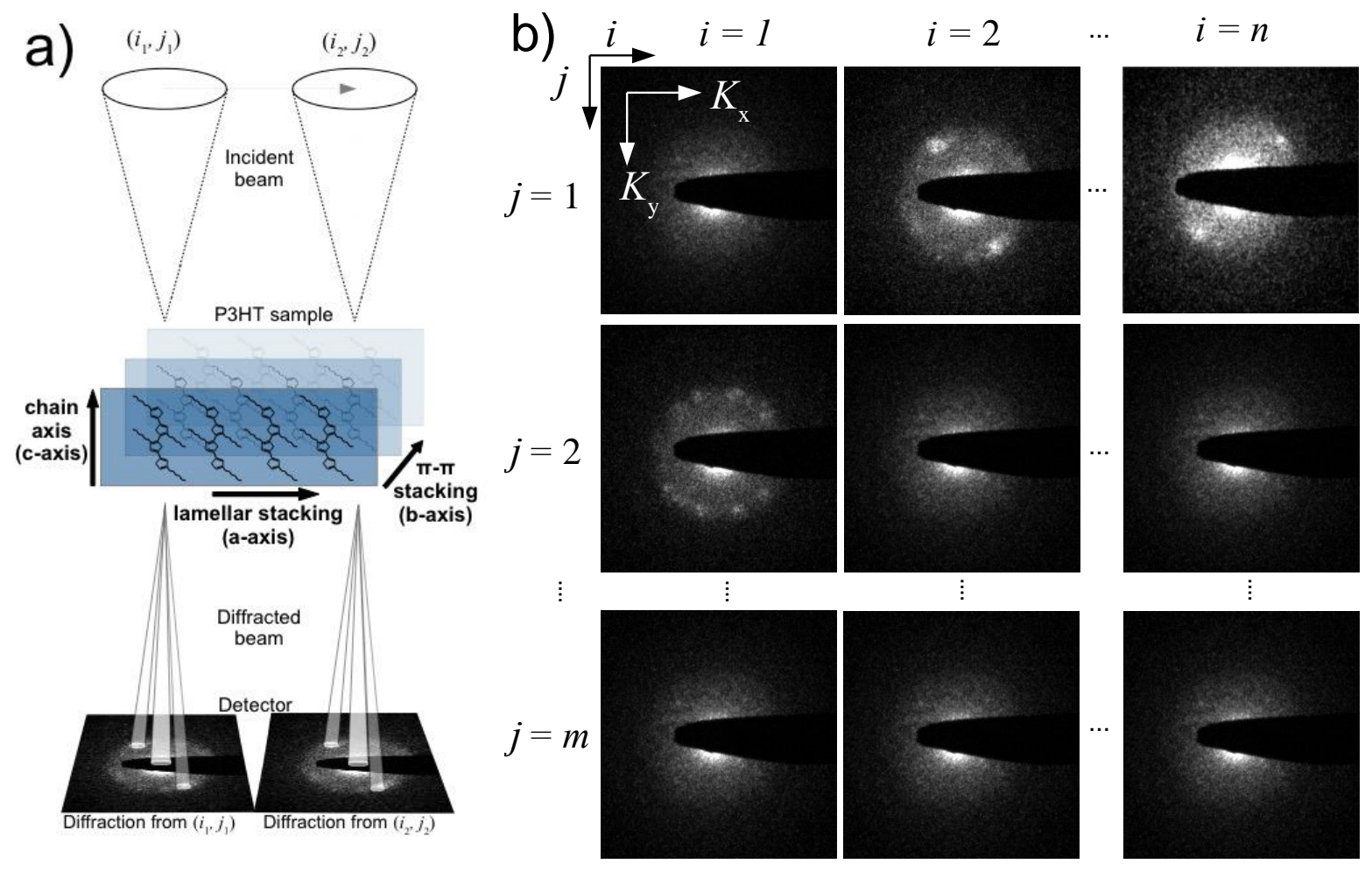

Figure 1. The 4D-STEM experiment. (a) Convergent beam rasters the beam across the sample $(i, j)$ and captures a full diffraction pattern $\left(K_{\mathrm{x}}, K_{\mathrm{y}}\right)$ for each probe location. (b) The data takes the form of a 4-dimensional $n \times m$ set where each pixel in real space $(i, j)$ corresponds to a full diffraction pattern $\left(K_{\mathrm{x}}, K_{\mathrm{y}}\right)$.

The stacks of DPs obtained during each scan were subsequently mined to reveal the distribution of the crystallites. Each DP in the set corresponds to a real space position $(i, j)$ of the rastered beam; crystallite orientation parameters can be extracted by locating and indexing the Bragg diffraction spots. While a single diffraction pattern can be manually indexed, 4D-STEM can raster areas as large as $128 \mathrm{x}$ 128 probe positions, resulting in 16384 individual diffraction patterns that require automated analysis routines.

\section{b. Electron Dose considerations}

Electron beam damage in polymers is ubiquitous, and several recent review articles address this issue (Egerton, 2012; Libera and Egerton, 2010). Experimentally, we observe the damage as a rapid disappearance of diffraction spots; changes in accelerating voltage between 200 and $300 \mathrm{kV}$ did not show any observable difference in diffraction persistence. Careful studies of diffraction intensity as a function of time can result in a characteristic dose, $D_{e}$, for a given material (Egerton et al., 2012; Siangchaew and Libera, 2009); these measurements were not carried out here.

Often the term "low-dose" electron microscopy is used to describe parallel beam TEM with an electron dose on the order of $10 \mathrm{e}^{-} / \AA^{2}$. Because this is a convergent beam technique and all the electrons 
are focused to a small area $\left(\sim 4000 \AA^{2}\right)$, the electron dose rate is high at $\sim 8000 \mathrm{e}^{-} / \AA^{2} \cdot \mathrm{s}$. The short exposure time of $70 \mathrm{~ms}$ reduces the dose to $\sim 600 \mathrm{e}^{-} / \AA^{2}$, and one can decrease the dose even further by defocusing the probe over a larger area.

Measurement of the probe size was done by imaging the convergent beam at high magnification onto a charge-coupled device. A profile of the probe image at $200 \mathrm{kV}$ shows a shape with a full-width half-maximum (FWHM) of $7 \mathrm{~nm}$ and a dose of $\sim 2000 \mathrm{e}^{-} / \AA^{2}$ at its peak. The total beam current was measured at $5 \mathrm{pA}$, which corresponds to an average electron dose of $8000 \mathrm{e}^{-} / \AA^{2}$, over an area with a diameter defined by the FWHM of $7 \mathrm{~nm}$. Ten nanometers from the center of the probe the dose is still on the order of $20 \mathrm{e}^{-} / \AA^{2}$. This data shows that not all parts of the sample receive an equivalent dose; the optimal probe shape for diffraction studies of polymers remains to be investigated.

The impact of this technique is the ability to step to a new area of the sample that has not yet been exposed to electrons. The diffraction pattern is robust against changes in focus conditions so one does not need to expose an area to achieve a focus condition. Additionally, diffraction is efficient at using electrons, and contrast in a diffraction pattern is much easier to achieve than in a conventional TEM image. The short time scales used effectively eliminate errors and distortions that could be incurred by stage vibrations or sample charging. By keeping track of the history of the stage motions, one can step to a new area of the sample with the beam blocked and then acquire a dataset with the possibility of capturing an interesting area. In this work we keep track of the exposure in an effort to prevent the sample from altering its structure.

\section{Results and Discussion}

\section{a. Analysis Algorithm}

Methods for manual and automated analysis of diffraction patterns with high signal/noise have been reported previously (Gammer et al., 2015; Henderson and Glaeser, 1985; Ozdol et al., 2015); however, automated analysis of DP sets from semicrystalline polymers, such as those explored here, requires additional steps. In soft materials Bragg reflections from crystalline features have weak intensities due to the low electron dose. Additionally, they are superimposed onto a monotonically decaying background (Figure $2 b$ ), which varies in intensity from one probe position to the next. We propose that this background arises from interactions between the incident beam and amorphous regions in the sample (amorphous halo). Furthermore, the beam tilts as it rasters the sample, which causes the diffraction patterns to shift within the detector's field of view. This causes the patterns to be misaligned non-linearly with respect to one another within the dataset (Figure 2a); this effect is greater at lower magnifications. The beamstop, necessary for image sensor protection, hides the central transmitted beam and eliminates a point of reference that could have been used to align the patterns to one another.
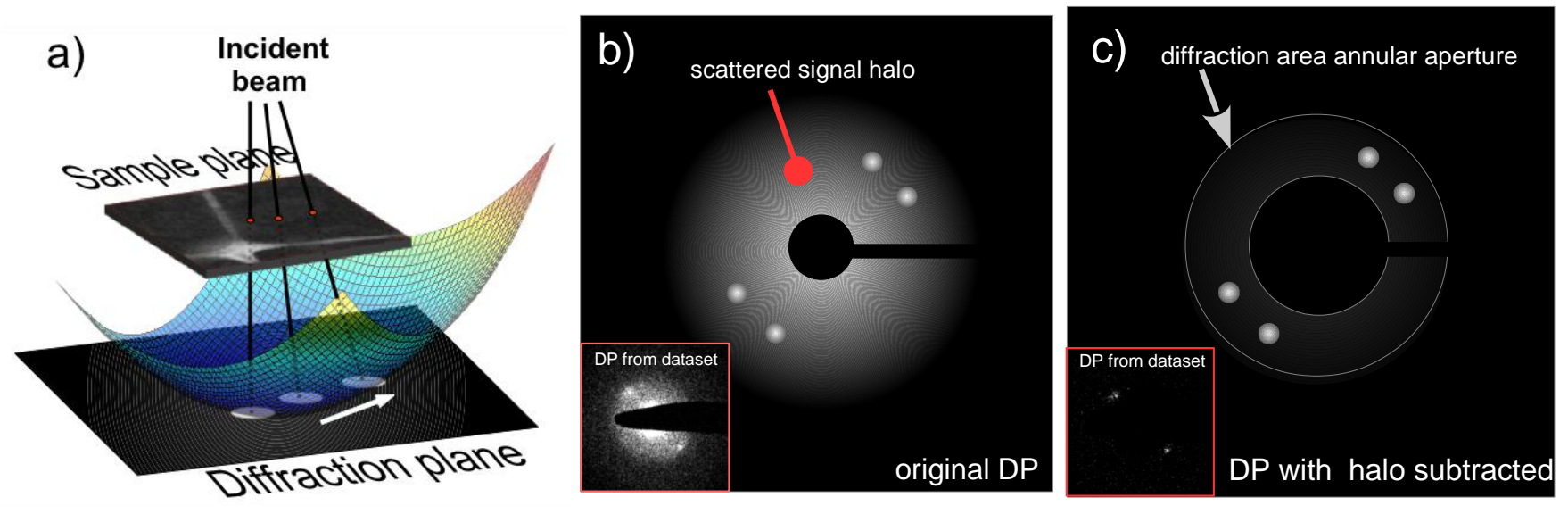
Figure 2. Schematic illustration of the algorithm developed. (a) Diagram of the effect of the beam sway as it scans the sample; the colored surface is a parabolic fit to calculated beam shifts for the P3HT sample dataset used in this study. (b) Diagram of a typical diffraction pattern obtained in 4D-STEM, with diffraction spots superimposed on a background scattered halo. Inset shows a DP from a gathered dataset. (c) Diffraction pattern after processing, with the background halo removed and the diffraction diffraction spots clearly featured. A virtual annular aperture used for virtual dark field calculations is outlined in white. Inset shows the DP from (b) after application of the algorithm.

The first step in the algorithm is to align the DPs. The true center of each diffraction pattern is found by fitting an ellipse to a subset of points taken from the amorphous halo and calculating its center of mass. The patterns are then shifted such that the calculated center matched the center point of the detector's field of view (Figure 2a).

The second step is to remove the amorphous halo; a different amorphous halo is subtracted from each DP. First, the averaged intensity of the scattered amorphous background is computed using an aperture that excludes the ring where diffraction occurs, obtaining an amorphous scale factor $h_{\mathrm{i}, \mathrm{j}}$ for each probe location $(i, j)$. The matrix $h_{\mathrm{i}, \mathrm{j}}$ is then normalized by a constant corresponding to the largest value of $h_{\mathrm{i}, \mathrm{j}}$. A reference halo image $I_{\mathrm{h}}\left(K_{\mathrm{x}}, K_{\mathrm{y}}\right)$ is generated by summing all of the diffraction patterns and normalizing by the number of probe positions. The intensity values of the reference halo image are then multiplied by $2 h_{\mathrm{i}, \mathrm{j}}$ to obtain a reference halo tailored to each probe position $(i, j)$; the factor of 2 allows for an efficient suppression of the amorphous signal. The specific background halo image is then subtracted from its respective DP. Negative pixel values resulting from the subtraction are set to zero. This effectively decouples the diffraction signal from the scattered halo surrounding it. The halo subtraction procedure is illustrated schematically in Figures $2 b$ and $2 c$; typical data before and after subtraction are shown in the insets. After the correction, all DPs exhibiting no Bragg diffraction are reduced to flat, near-zero images.

The third step is to generate virtual dark field images by summing the signal from each DP that falls within a defined aperture area. This step is equivalent to the experimental use of an objective aperture selecting given diffraction conditions (Sherman et al., 1981); in the case of soft materials, the ability to obtain all possible dark field images with a single exposure eliminates damage that would be caused by multiple exposures. Of course, virtual dark field images can also be obtained by summing the total intensity at each probe location without any processing.

Finally, the positions of the diffracted spots can be found by locating peaks of highest intensity whose centers fall within a narrow range of distances from the center, as defined by the polymer's observable Bragg reflections and achieved via a virtual annular aperture (Figure 2c). Acquired diffraction frames were of three types: those with only one Bragg reflection present per frame, those with more than one Bragg reflection per frame, and those with no Bragg diffraction present. Diffraction patterns containing more than one crystal orientation occur either because the beam has sampled adjacent crystallites or because there are layers of crystalline material; the multiple orientations are twists about the axis parallel to the beam. Frames with no visible diffraction are either not aligned or not crystalline. Some DPs exhibit bright diffraction disks while others can barely be distinguished. In this study, we conservatively accepted peaks as "visible" only if their integrated signal over the disk area was strong enough to outcompete peaks arising from background noise after halo subtraction. As the center of each DP is known, we can then easily compute the relative angle of the diffraction spots and create a Bragg diffraction angle map. 


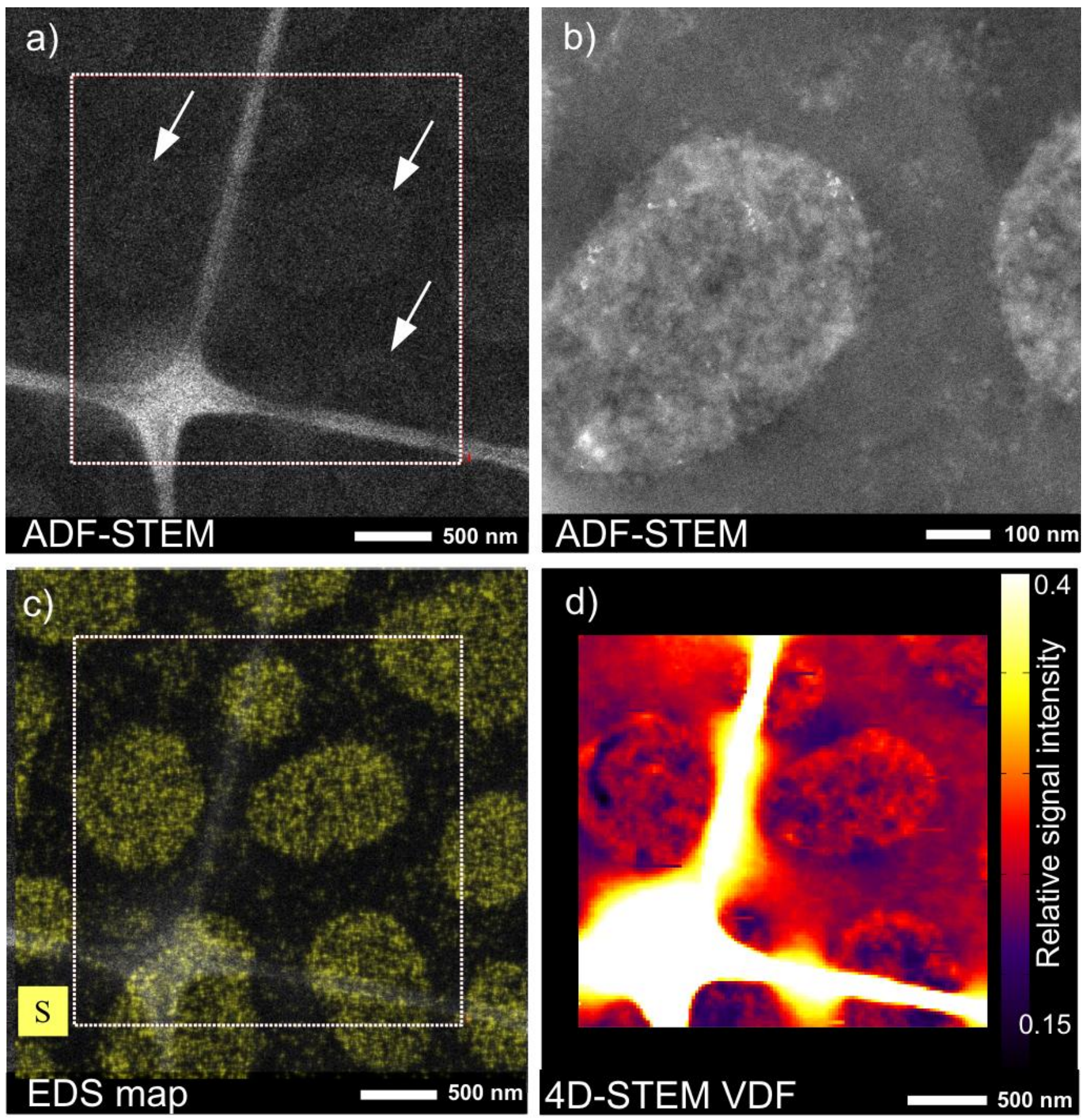

Figure 3. Raw and minimally processed images for P3HT/PSS (200 kV). (a) ADF-STEM image with oval clusters of P3HT shown by white arrows. (b) Aggregate at higher magnification displaying structure within. (c) The composition of the aggregates is confirmed to be mostly P3HT by an EDS map which shows cumulative sulfur signal in yellow. (d) From the 4D-STEM dataset gathered over the square region delineated by white dashed lines in (a) and (c), a preliminary virtual dark field image can be reconstructed using the entirety of the field of view. Color indicates the normalized relative cumulative signal intensity for each probe position. 


\section{b. Crystallinity of P3HT/PS}

Figure $3 \mathrm{a}, 3 \mathrm{~b}$ and $3 \mathrm{c}$ show images of the P3HT/PS sample captured at an accelerating voltage of $200 \mathrm{kV}$. Under annular dark field (ADF) STEM mode, large ( $200 \mathrm{~nm}$ ) oval disks were observed (Figure $3 a)$; when surveyed at higher magnification they were shown to exhibit rough internal features (Figure 3b). Sulfur EDS confirmed that these structures were mostly P3HT (Figure 3c).

A virtual dark field image with an aperture that includes the whole field of view of the detector is shown in Figure 3d, prior to alignment or halo subtraction. This image contains signal from both the diffracting and amorphous regions and provides a measure of the relative thickness of the sample, which shows structure that was not discernible under ADF-STEM conditions. The lacey carbon support scatters electrons more intensely than the polymer sample and thus appears saturated on the image due to the very bright background halo it generates.

The results of our analysis on the P3HT/PS sample are presented in Figures 4 and 5. Figure 4a is a ADF-STEM image of an independent P3HT/PS blend taken at $300 \mathrm{kV}$. In some experiments, the P3HTrich oval disks appeared brighter than the background (Figure 3b), while in others they appeared darker (Figure 4a). The origin of this difference in contrast is presumably due to a thickness differential in the PS film surrounding the oval structures. The use of different apertures allows us to probe different aspects of the sample morphology, as exemplified in Figures 4b-c. The virtual dark field image in Figure $4 \mathrm{~b}$ is constructed before subtraction of the background halo; we can see that without halo subtraction or use of a virtual aperture around the diffraction locations, the scattered amorphous signal dominates the image. When our algorithm is employed, however, the on-axis crystalline domains are immediately distinguishable. (Figure 4c).
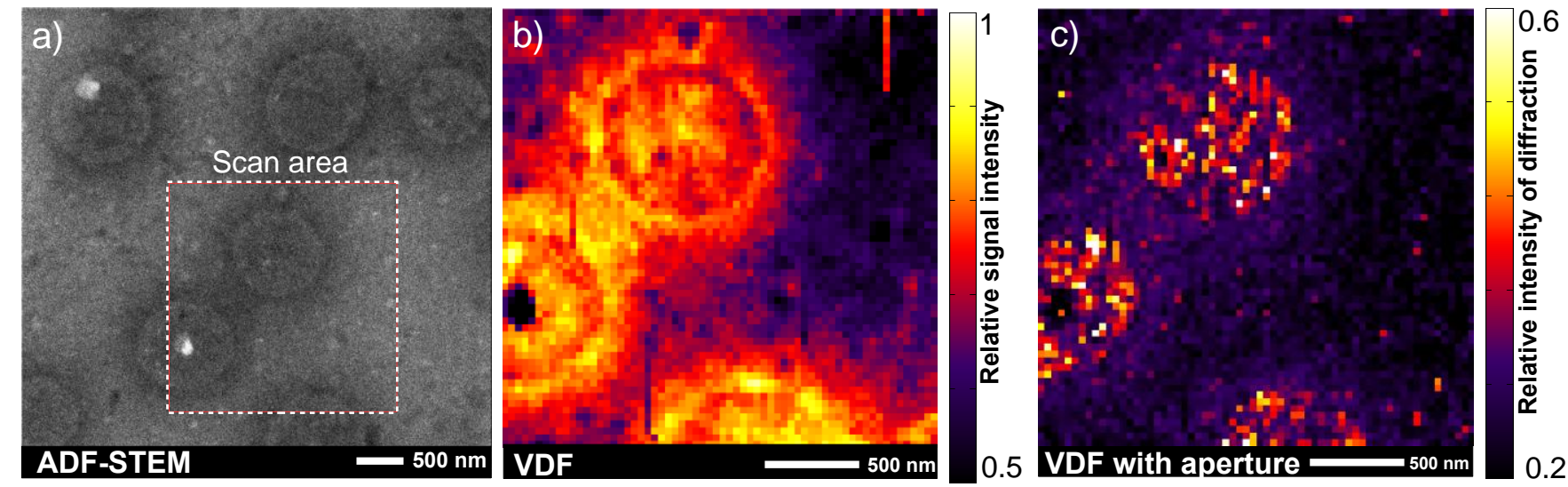

Figure 4. Algorithm applied to P3HT/PS sample at $300 \mathrm{kV}$. (a) ADF-STEM image of P3HT sample, with the 4D-STEM-rastered region outlined in a white dashed square. Two VDFs of the 4D-STEM dataset (step size of $40 \mathrm{~nm}$ ) are shown. (b) VDF image constructed from signal summed over the whole field of view of the detector. (c) VDF image constructed from halo-subtracted signal within a thin virtual annular aperture that only includes locations where diffraction peaks occur.

An aperture-aided virtual dark field, similarly constructed to the one shown in Figure 4c, is presented from a different sample in Figure 5a. The virtual dark field image is normalized and locations where crystalline reflections are strongest appear in white and yellow. These results clearly show that the oval structures are not exhibiting Bragg reflections over their entire area. The Bragg reflections that do appear on the DPs are shown in Figure 5c, with their scattering angle plotted in color and overlaid onto the ADF image. While a few of the crystalline reflections can be seen scattered throughout the space between the aforementioned large oval structures, most of the crystalline reflections seen are contained within those oval regions. Furthermore, clusters of adjacent pixels showing Bragg peaks with similar orientations can clearly be observed, and can span distances as large as $80 \mathrm{~nm}$. The distribution of the crystallite orientations mapped within the oval clusters appears to be fairly uniform, and the crystalline features are 
oriented randomly with respect to one another. Reflections around $90^{\circ}$ are seldom picked up by the algorithm, as they are masked by the beamstop, as illustrated in the colorwheel legend of Figure 5b. Finally, we can obtain a measure for the localized degree of crystallinity by computing the ratio between the now decoupled diffracted and amorphous signals. The diffracted signal is taken to be the VDF such as the one in Figures 4c and 5a, where only diffracted Bragg peaks appear as non-zero features. The amorphous signal is an $i$ by $j$ map of the amorphous scale factors $h_{\mathrm{i}, \mathrm{j}}$ obtained previously. This result is shown in Figure 5c and further confirms the location and distribution of P3HT crystalline features within the oval structures.
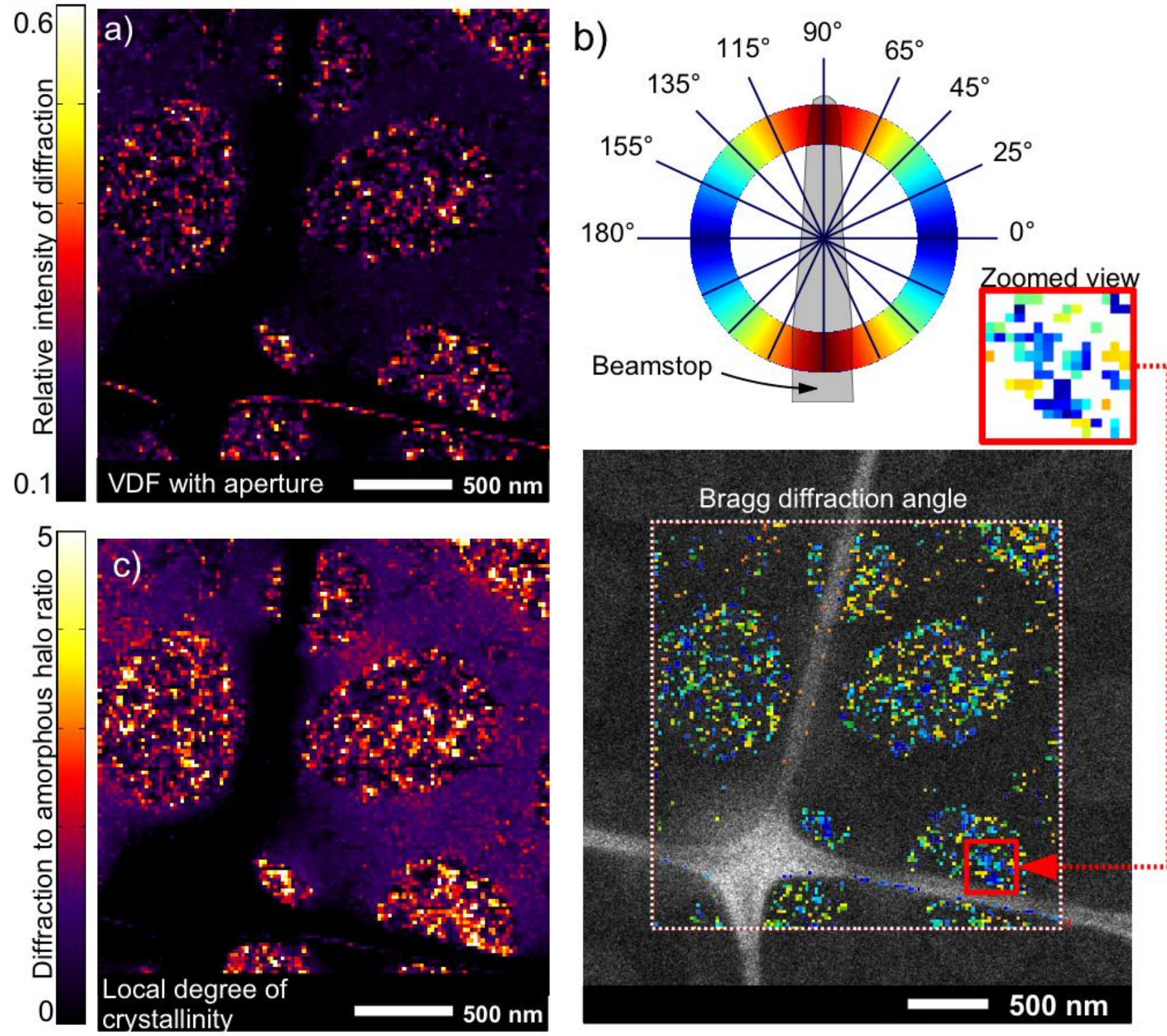

Figure 5. Algorithm applied to P3HT/PS sample at $200 \mathrm{kV}$. (a) Normalized VDF showing the relative intensity of the Bragg reflections, obtained from a processed dataset where the background scattered halo has been removed. (b) Map of a normalized local degree of crystallinity, as defined here by the ratio of the Bragg diffraction signal to the extracted amorphous scattered signal.

Yellow spots indicate high degree of crystallinity. (c) Bragg diffraction angle map of the rastered region, with the angle map overlaid onto the ADF image. The legend is shown above as a colorwheel; the beamstop is drawn to show the obscured range of reflections.

Measurements from individual diffraction patterns indicate that the P3HT polymer lamellae could be lying edge-on as well as face-on, as described by Brinkmann et al (Brinkmann and Rannou, 2007) for 
a low MW semicrystalline P3HT sample. Visual measurements of the Bragg reflections observed yielded an average interplanar distance of $3.87 \AA \pm 0.12 \AA$ over 41 patterns; conversely, the same measurement was made using the Bragg reflections found by the algorithm, which resulted in a value of $3.71 \AA \pm 0.29 \AA$ over 1805 patterns. The error is larger for the algorithm due to the non-uniform illumination of the Bragg spots, which leads to error in the estimation of the true center of the peak. This measured periodicity at $\sim 3.8 \AA$ agrees well with the $3.9 \AA$ stacking period of successive polythiophene backbones along the $b$-axis of a monoclinic structure modeled by Kayunkid et al (Kayunkid et al., 2010) and observed by others (Brinkmann and Rannou, 2007; Joshi et al., 2008; Prosa et al., 1992; Tashiro et al., 1997). This reflection corresponds to the (020) reflection of the monoclinic structure proposed by Joshi (Joshi et al., 2008) and Brinkmann (Brinkmann and Rannou, 2007), or the (020) reflection of the orthorhombic structure proposed by Tashiro (Tashiro et al., 1997) and Prosa (Prosa et al., 1992). This periodicity corresponds to the $\pi-\pi$ bond spacing and would appear on the DP only if the crystallite is oriented edge-on, with either the $a$ - or $c$-axis parallel to the beam (Kline et al., 2006). This spacing, however, is extremely close to the (002) reflection characteristic of monomer spacing along the backbone (3.88 $\AA$ ) (Brinkmann and Rannou, 2007) that would be exhibited by face-on oriented crystallites, to the point that we cannot distinguish between the two. Furthermore, for a film thickness of approximatively $30 \mathrm{~nm}$ the ratio of edge-on to face-on orientations is estimated to be $\sim 1$ according to Huang et al (Huang et al., 2012), which means that both reflections are equally likely to occur. Diffraction spots generated by longer stacking distances - and thus appearing closer to the center in diffraction space - while present within the signal, are completely masked by the central transmitted beam and therefore could not be extracted.

If one of the axes $p$ of a crystallite is parallel to the electron beam, diffraction spots indicate that its orthogonal axis is randomly oriented in twists about $p$. The Bragg rotation map shown in Figure 5c shows that crystallites can be bigger than our step size of $20 \mathrm{~nm}$, with slight misalignments across adjacent probe positions. This is expected; indeed, in addition to a low signal to noise ratio of the diffracted signal, which makes some peak locations ambiguous, polymer chains could show a propensity to bend even when in a semi-crystalline state. This would lead not only to a slight rotation of the Bragg peaks from one pattern to the next, but also to the disappearance of others as the lattice planes twist and bend out of axis alignment. This would indicate that the crystalline regions mapped here are only a subset of larger and/or more numerous crystalline features, which are not accessible through 4D-STEM unless the sample is tilted into the corresponding Bragg conditions. Therefore, dark areas on the virtual dark fields do not necessarily indicate amorphous regions, although those are bound to be present as well. While not investigated in this study, these ambiguous regions could be characterized with a combination of tilting experiments and better dose control.

\section{Conclusion}

We have introduced a scanning transmission electron microscopy technique to obtain spatially resolved maps of crystalline domain orientations over a field of view on the order of 1 micron with a spatial resolution of $20 \mathrm{~nm}$. Corrections for beam tilt and amorphous scattering were essential for obtaining these maps. This technique was applied to a blend of P3HT and PS with containing P3HT-rich oval shaped domains. Regions on order $80 \mathrm{~nm}$ in length appeared to have coherently oriented crystals. Such maps may prove useful for determining pathways for electronic charge transport in P3HT-containing materials. The technique we have developed, however, is perfectly general, and can be applied to any semicrystalline polymer sample.

\section{Acknowledgments}

Primary funding for the work was provided by the Electron Microscopy of Soft Matter Program from the Office of Science, Office of Basic Energy Sciences, Materials Sciences and Engineering Division of the U.S. Department of Energy under Contract No. DE-AC02-05CH11231. The electron 
microscopy was performed as a user project at the Molecular Foundry at Lawrence Berkeley National Laboratory, which is supported by the U.S. Department of Energy under Contract \# DE-AC02$05 \mathrm{CH} 11231$. 


\section{References}

Bhatt, M.P., Thelen, J.L., Balsara, N.P., 2015. Effect of Copolymer Composition on Electronic Conductivity of Electrochemically Oxidized Poly(3-hexylthiophene)-b-poly(ethylene oxide) Block Copolymers. Chemistry of Materials 27, 5141-5148.

Brinkmann, M., Rannou, P., 2007. Effect of Molecular Weight on the Structure and Morphology of Oriented Thin Films of Regioregular Poly(3-hexylthiophene) Grown by Directional Epitaxial Solidification. Advanced Functional Materials 17, 101-108.

Chan, K., Yamao, T., Kotaki, M., Hotta, S., 2010. Unique structural features and electrical properties of electrospun conjugated polymer poly(3-hexylthiophene) (P3HT) fibers. Synthetic Metals 160, 2587-2595.

Chu, C.-W., Yang, H., Hou, W.-J., Huang, J., Li, G., Yang, Y., 2008. Control of the nanoscale crystallinity and phase separation in polymer solar cells. Applied Physics Letters 92, 103306.

Coropceanu, V., Cornil, J., da Filho, D.A., Olivier, Y., Silbey, R., Bredas, J.L., 2007. Charge Transport in Organic Semiconductors. ChemInform 38, 926-952.

da Silva, M.A.V., Mortimer, C.T., 1984. Thermochemistry and Its Applications to Chemical and Biochemical Systems. springer, 47-60.

Dag, S., Wang, L.-W., 2010. Packing structure of poly(3-hexylthiophene) crystal: ab initio and molecular dynamics studies. The journal of physical chemistry. B 114, 5997-6000.

Dudenko, D., Kiersnowski, A., Shu, J., Pisula, W., Sebastiani, D., Spiess, H., Hansen, M., 2012. A Strategy for Revealing the Packing in Semicrystalline $\pi$-Conjugated Polymers: Crystal Structure of Bulk Poly-3-hexyl-thiophene (P3HT). Angewandte Chemie International Edition 51, 11068-11072.

Duong, D.T., Wang, C., Antono, E., Toney, M.F., Salleo, A., 2013. The chemical and structural origin of efficient p-type doping in P3HT. Organic Electronics 14, 1330-1336.

Gammer, C., Ozdol, B.V., Liebscher, C.H., Minor, A.M., 2015. Diffraction contrast imaging using virtual apertures. Ultramicroscopy 155, 1-10.

Hammer, B.A.G., Reyes-Martinez, M.A., Bokel, F.A., Liu, F., Russell, T.P., Hayward, R.C., Briseno, A.L., Emrick, T., 2014. Robust polythiophene nanowires cross-linked with functional fullerenes. Journal of Materials Chemistry C 2, 9674-9682.

Henderson, R., Glaeser, R.M., 1985. Quantitative analysis of image contrast in electron micrographs of beam-sensitive crystals. Ultramicroscopy 16, 139-150.

Huang, B., Glynos, E., Frieberg, B., Yang, H., Green, P.F., 2012. Effect of thickness-dependent microstructure on the out-of-plane hole mobility in poly(3-hexylthiophene) films. ACS applied materials \& interfaces 4, 5204-5210.

Joshi, S., Grigorian, S., Pietsch, U., 2008. X-ray structural and crystallinity studies of low and high molecular weight poly(3-hexylthiophene). physica status solidi (a) 205, 488-496.

Kadem, B.Y., Al-hashimi, M.K., Hassan, A.K., 2014. The Effect of Solution Processing on the Power Conversion Efficiency of P3HT-based Organic Solar Cells. Energy Procedia 50, 237-245. 
Kao, K.-Y., Lo, S.-C., Chen, H.-L., Chen, J.-H., Chen, S.-A., 2014. Gelation of a Solution of Poly(3-hexylthiophene) Greatly Retards Its Crystallization Rate in the Subsequently Cast Film. The Journal of Physical Chemistry B 118, 14510-14518.

Kayunkid, N., Uttiya, S., Brinkmann, M., 2010. Structural Model of Regioregular Poly(3hexylthiophene) Obtained by Electron Diffraction Analysis. Macromolecules 43, 4961-4967.

Kline, J.R., McGehee, M.D., Toney, M.F., 2006. Highly oriented crystals at the buried interface in polythiophene thin-film transistors. Nature Materials 5, 222-228.

Ludwigs, S., Poelking, C., Daoulas, K., Troisi, A., Andrienko, D., 2014. Advances in Polymer Science. springer, 139-180.

Mårdalen, J., Samuelsen, E.J., Gautun, O.R., Carlsen, P.H., 1992. X-ray scattering from oriented poly(3-alkylthiophenes). Synthetic Metals 48, 363-380.

Mazzio, K.A., Luscombe, C.K., 2014. The future of organic photovoltaics. Chemical Society Reviews 44, 78-90.

McMahon, D.P., Cheung, D.L., Goris, L., Dacuña, J., Salleo, A., Troisi, A., 2011. Relation between Microstructure and Charge Transport in Polymers of Different Regioregularity. The Journal of Physical Chemistry C 115, 19386-19393.

Meredig, B., Salleo, A., Gee, R., 2009. Ordering of Poly(3-hexylthiophene) Nanocrystallites on the Basis of Substrate Surface Energy. ACS Nano 3, 2881-2886.

Motaung, D.E., Malgas, G.F., Arendse, C.J., 2010. Comparative study: The effects of solvent on the morphology, optical and structural features of regioregular poly(3-hexylthiophene):fullerene thin films. Synthetic Metals 160, 876-882.

Müller, C., Radano, C.P., Smith, P., Stingelin-Stutzmann, N., 2008. Crystalline-crystalline poly(3-hexylthiophene)-polyethylene diblock copolymers: Solidification from the melt. Polymer 49, 3973-3978.

Northrup, J.E., 2007. Atomic and electronic structure of polymer organic semiconductors: P3HT, PQT, and PBTTT. Physical Review B 76.

Ozdol, V.B., Gammer, C., Jin, X.G., Ercius, P., Ophus, C., Ciston, J., Minor, A.M., 2015. Strain mapping at nanometer resolution using advanced nano-beam electron diffraction. Applied Physics Letters 106, 253107.

Paquin, F., Rivnay, J., Salleo, A., Stingelin, N., Silva-Acuña, C., 2015. Multi-phase microstructures drive exciton dissociation in neat semicrystalline polymeric semiconductors. Journal of Materials Chemistry C 3, 10715-10722.

Park, S.-Y., Moon, S.-C., Dang, T.D., Venkatasubramanian, N., Lee, J.-w., Farmer, B.L., 2005. The crystal structure of poly(2,6-naphthalenebenzobisthiazole). Polymer 46, 5630-5636.

Perlich, J., Rubeck, J., Botta, S., Gehrke, R., Roth, S.V., Ruderer, M.A., Prams, S.M., Rawolle, M., Zhong, Q., Körstgens, V., Müller-Buschbaum, P., 2010. Grazing incidence wide angle X-ray scattering at the wiggler beamline BW4 of HASYLAB. The Review of scientific instruments 81, 105105.

Prosa, T.J., Winokur, M.J., Moulton, J., Smith, P., Heeger, A.J., 1992. X-ray structural studies of poly(3-alkylthiophenes): an example of an inverse comb. Macromolecules 25, 4364-4372. 
Randle, V., 2009. Recent Developments in Electron Backscatter Diffraction. Advances in Imaging and Electron Physics 151, 363-416.

Remy, R., Weiss, E., Nguyen, N.A., Wei, S., Campos, L.M., Kowalewski, T., Mackay, M.E., 2014. Enthalpy of fusion of poly(3-hexylthiophene) by differential scanning calorimetry. Journal of Polymer Science Part B: Polymer Physics 52, 1469-1475.

Sherman, E.S., Adams, W.W., Thomas, E.L., 1981. Dark field imaging of semicrystalline polymers by scanning transmission electron microscopy. Journal of Materials Science 16, 1-9.

Siangchaew, K., Arayasantiparb, D., Libera, M., 1996. Measuring Polymer Microstructure using Spatially-Resolved Eels in the Stem. MRS Proceedings 461.

Takacs, C.J., Collins, S.D., Love, J.A., Mikhailovsky, A.A., Wynands, D., Bazan, G.C., Nguyen, T.-Q., Heeger, A.J., 2014. Mapping Orientational Order in a Bulk Heterojunction Solar Cell with Polarization-Dependent Photoconductive Atomic Force Microscopy. ACS Nano 8, 8141-8151.

Takacs, C.J., Treat, N.D., Krämer, S., Chen, Z., Facchetti, A., Chabinyc, M.L., Heeger, A.J., 2013. Remarkable Order of a High-Performance Polymer. Nano Letters 13, 2522-2527.

Tashiro, K., Kobayashi, M., Kawai, T., Yoshino, K., 1997. Crystal structural change in poly(3alkyl thiophene)s induced by iodine doping as studied by an organized combination of X-ray diffraction, infrared/Raman spectroscopy and computer simulation techniques. Polymer 38, 2867-2879.

Wirix, M.J.M., Bomans, P.H.H., Friedrich, H., Sommerdijk, N.A.J.M., de With, G., 2014. ThreeDimensional Structure of P3HT Assemblies in Organic Solvents Revealed by Cryo-TEM. Nano Letters 14, 2033-2038.

Xiao, X., Wang, Z., Hu, Z., He, T., 2010. Single Crystals of Polythiophene with Different Molecular Conformations Obtained by Tetrahydrofuran Vapor Annealing and Controlling Solvent Evaporation. The Journal of Physical Chemistry B 114, 7452-7460.

Xie, Y., Dutta, P., Cengher, D., Bommisetty, V., Li, J., Galipeau, D., Qiao, Q., 2009. Solvent effect on the morphology of P3HT/PCBM films. spie.

Yazawa, K., Inoue, Y., Shimizu, T., Tansho, M., Asakawa, N., 2010. Molecular Dynamics of Regioregular Poly(3-hexylthiophene) Investigated by NMR Relaxation and an Interpretation of Temperature Dependent Optical Absorption. The Journal of Physical Chemistry B 114, 12411248. 